\title{
Glucose-installed, SPIO-loaded PEG- $b$-PCL micelles as MR contrast agents to target prostate cancer cells
}

\author{
Man Theerasilp ${ }^{1,2} \cdot$ Panya Sunintaboon $^{3} \cdot$ Witaya Sungkarat $^{4} \cdot$ Norased Nasongkla $^{1,2}$
}

Received: 24 March 2017 / Accepted: 22 September 2017/Published online: 10 October 2017

(c) The Author(s) 2017. This article is an open access publication

\begin{abstract}
Polymeric micelles of poly(ethylene glycol)block-poly( $\varepsilon$-caprolactone) bearing glucose analog encapsulated with superparamagnetic iron oxide nanoparticles (Glu-SPIO micelles) were synthesized as an MRI contrast agent to target cancer cells based on high-glucose metabolism. Compared to SPIO micelles (non-targeting SPIO micelles), Glu-SPIO micelles demonstrated higher toxicity to human prostate cancer cell lines (PC-3) at high concentration. Atomic absorption spectroscopy was used to determine the amount of iron in cells. It was found that the iron in cancer cells treated by Glu-SPIO micelles were 27 -fold higher than cancer cells treated by SPIO micelles at the iron concentration of $25 \mathrm{ppm}$ and fivefold at the iron concentration of $100 \mathrm{ppm}$. To implement Glu-SPIO micelles as a MR contrast agent, the 3-T clinical MRI was applied to determine transverse relaxivities $\left(r_{2}{ }^{*}\right)$ and relaxation rate $\left(1 / T_{2} *\right)$ values. In vitro MRI showed different MRI signal from cancer cells after cellular uptake of SPIO micelles and Glu-SPIO micelles. Glu-SPIO micelles
\end{abstract}

Norased Nasongkla

norased.nas@mahidol.ac.th

1 Department of Biomedical Engineering, Faculty of Engineering, Mahidol University, 25/25 Puttamonthon 4th Rd, Nakorn Pathom 73170, Thailand

2 Department of Chemistry and Center of Excellence for Innovation in Chemistry, Faculty of Science, Mahidol University, Bangkok 10400, Thailand

3 Department of Chemistry, Faculty of Science, Mahidol University, Nakorn Patom 73170, Thailand

4 Department of Radiology, Faculty of Medicine, Ramathibodi Hospital, Mahidol University, Bangkok 10400, Thailand was highly sensitive with the $r_{2} *$ in agarose gel at $155 \mathrm{mM}^{-1} \mathrm{~s}^{-1}$. Moreover, the higher $1 / T_{2} *$ value was found for cancer cells treated with Glu-SPIO micelles. These results supported that glucose ligand increased the cellular uptake of micelles by PC-3 cells with over-expressing glucose transporter on the cell membrane. Thus, glucose can be used as a small molecule ligand for targeting prostate cancer cells overexpressing glucose transporter.

Keywords Drug delivery system · Polymeric micelles . Superparamagnetic iron oxide $\cdot$ MR contrast agents . Targeting nanoparticles

\section{Introduction}

Imaging techniques are non-invasive methods utilized to diagnose cancer in human body. However, the challenging tasks still persist where improvement is needed to overcome limitations. Magnetic resonance imaging (MRI) provides higher resolution for anatomic imaging purposes but less molecular and physiological information (Hoffman and Gambhir 2007; Weissleder and Pittet 2008). ${ }^{18}$ F-fluoro-2-deoxyglucose positron emission tomography $\left({ }^{18} \mathrm{~F}\right.$-FDG PET) has been used successfully for assessing state of tumors, planning and monitoring of tumor therapy as well as the early detection of recurrent tumor growth. 2-[18F]-2-deoxy-D-glucose $\left({ }^{18} \mathrm{~F}-\mathrm{FDG}\right)$, a glucose analog molecule, is taken up by cells via the facilitated glucose transporter, especially glucose transporter 1 (Glut-1). It is accumulated within cells in direct proportion to their metabolic activity. Thus, the high uptake of ${ }^{18}$ F-FDG by tumor cells can be detected by PET scan. However, ${ }^{18}$ F-FDG is a radio-labeling 
material which can cause potential hazards to patients and presents a limited half-life. Moreover, PET has very low resolution and expensive. To overcome these limitations, the targeting capability of Glut-1 can be adopted to target cancer cells using other imaging techniques such as MRI. MRI is a good candidate to be applied as the molecular imaging due to high-resolution and high soft-tissue contrast. Currently, gadolinium and superparamagnetic iron oxide (SPIO) are widely used as MRI contrast agents and were used to enhance MR image contrast for clinical applications (Jin et al. 2014; Liu et al. 2016). In addition, superparamagnetic iron oxide nanoparticle was also applied for drug delivery system (Sheikholeslami 2017c). Many research works have reported properties of magnetic nanoparticles (Sheikholeslami 2017b) and studied magnetic field affecting nanoparticles (Sheikholeslami 2017a, c). Nanoparticles have been studied to target high-glucose metabolism of cancer cells. Luciani et al. synthesized PEGylated paramagnetic niosome with glucose labeling on the surface. The gadobenate dimeglumine was loaded into inner noisome vesicles. The particles showed significantly improved tumor targeting in human carcinoma tumor xenograft (Luciani et al. 2004). Xiong et al. synthesized 2-deoxy-D-glucose-modified SPIO particles to target Glut-1-overexpressing breast tumor (MDA-MB231) (Xiong et al. 2012). The $T_{2}$ signal intensity of MDA-MB-231 after treated with glucose-modified SPIO decreased significantly. Venturelli et al. demonstrated that glucose-coated magnetic nanoparticles could be applied for metabolic-based assays to detect cancer cells (Venturelli et al. 2016).

Polymeric micelles have been developed for nanoscale drug delivery system, diagnostic imaging and biomedical applications (Blanco et al. 2009; Nasongkla et al. 2006; Theerasilp et al. 2017) and clinical practice (Caster et al. 2016). Polymeric micelles can be prepared from biocompatible amphiphilic block copolymers, forming core-shell architecture. The hydrophobic core acts as a reservoir for water-insoluble compounds resulting in the significant increase in solubility and stability of these compounds (Puntawee et al. 2016). The hydrophilic shell protects polymeric micelles from mononuclear phagocytic system (MPS) (Jokerst et al. 2011), and reduces renal clearance (Movassaghian et al. 2015). These properties contribute to long blood circulation time as well as high bioavailability. Small size with narrow distribution and long circulation time of polymeric micelles provide the accumulation of polymeric micelles in tumor tissues by the enhanced permeability and retention (EPR) effect (Jokerst et al. 2011). Targeting polymeric micelles can be achieved via attaching the ligand on the surface of micelles which recognizes tumor-specific receptors. RGD (Nasongkla et al. 2004; Zhang et al. 2012) and folic acid (Cheng et al. 2011) are effective ligands for targeting cancer. The targeting strategy increased accumulation at tumor site and increased the uptake by cancer cells. In the addition, polymeric micelles which have different abilities such as targeting, therapy and diagnosis in a single particle were synthesized as multifunctional polymeric micelles. Nasongkla et al. developed multifunctional micellar platform by conjugating cRGD to polymeric micelles after the SPIO and doxorubicin were loaded in core of micelles. They found 2.5 folds greater accumulation in SLK cells and higher growth inhibition including darker $T_{2}$-weighted image compared to cRGDfree micelles (Nasongkla et al. 2006).

The aim of this study was to construct the targeting polymeric micelles containing SPIO. The surface of micelles was conjugated with analogous glucosamine, glucose, to target prostate cancer line (PC-3) which overexpresses Glut-1 receptor (Effert et al. 2004). The characterization and cytotoxicity of Glu-SPIO micelles were investigated. The amount of SPIO in cancer cells after cellular uptake was evaluated by atomic absorption spectroscopy. $T_{2}{ }^{*}$ and $1 / T_{2} *$ values of PC-3 cells were compared after incubated with Glu-SPIO micelles or SPIO micelles.

\section{Materials and methods}

\section{Materials and methods}

Ethylene oxide was obtained from Wintech service (Thailand). $\varepsilon$-Caprolactone ( $\varepsilon$-CL) was purchased from SigmaAldrich and purified by vacuum distillation over calcium hydride $\left(\mathrm{CaH}_{2}\right)$. 3-Buten-1-ol was purchased from Acros and purified by vacuum distillation over calcium hydride $\left(\mathrm{CaH}_{2}\right) .18$-Crown-6 was purchased from Acros and vacuum-dried overnight at $46{ }^{\circ} \mathrm{C}$. Mercaptopropionic acid and azobisisobutyronitrile (AIBN) were purchased from Sigma-Aldrich. Hydrophobic SPIO was synthesized as previously described method (Sun et al. 2004). $N$-hydroxysuccinimide (NHS), $N$-ethyl- $N^{\prime}$-(3-dimethylaminopropyl) carbodiimide hydrochloride (EDC), 2-methoxyethylamine and Nuclear Fast Red were purchased from Sigma-Aldrich. Glucose triethylamine and potassium ferrocyanide were purchased from Acros Organics. All solvents were purchased from RCI Labscan as analytic grade. For the synthesis of polymer, tetrahydrofuran (THF), dimethylformamide (DMF) and toluene were dried by refluxing over a sodium-benzophenone and distilled under dried argon. 


\section{Synthesis of HOOC-PEG- $b$-PCL}

\section{Synthesis of allyl-PEG}

A three-neck round-bottom flask, anhydrous 3-benten-1-ol, of potassium naphthalide solution (1 molar equivalent), and 18-crown-6 (1.2 molar equivalent) were added into anhydrous THF under Ar stream. The flask was cooled in an ice bath, and then condensed ethylene oxide was transferred into flask by a cannula under Ar stream. The reaction was stirred for $24 \mathrm{~h}$ in the ice bath and $72 \mathrm{~h}$ in room temperature under $\mathrm{Ar}$ atmosphere. The crude polymer was collected by precipitation in diethyl ether. The powder of allyl-PEG was isolated by filtration and then washed with diethyl ether.

\section{Synthesis of allyl-PEG-b-PCL}

Dried allyl-PEG $(0.2 \mathrm{mmol})$ was dissolved in anhydrous toluene under $\mathrm{Ar}$ stream. Anhydrous $\varepsilon$-caprolactone ( $8.75 \mathrm{mmol})$ was added into the flask containing polymer solution. The flask was heated to $140{ }^{\circ} \mathrm{C}$ then few drops of $\mathrm{Sn}(\mathrm{Oct})_{2}$ was added under $\mathrm{Ar}$ stream. The solution was heated at $140{ }^{\circ} \mathrm{C}$ and stirred for $24 \mathrm{~h}$ under Ar atmosphere. The product was purified by redissolving in acetone and precipitated twice in diethyl ether.

\section{Synthesis of carboxyl-PEG-b-PCL}

3-Mercaptopropionic acid (20.0 molar equivalent) and AIBN (1.0 molar equivalent) were added into the solution of allyl-PEG- $b$-PCL in anhydrous DMF. The reaction mixture was stirred at $65^{\circ} \mathrm{C}$ for $24 \mathrm{~h}$ under Ar atmosphere.

\section{Preparation of SPIO-loaded micelles}

Micelles were prepared by solvent evaporation method (Theerasilp and Nasongkla 2013). Firstly, HOOC-PEG- $b$ PCL and SPIO were dissolved in THF and was added dropwise into DI water, while the mixture was sonicated continuously (SONIC, Model VCX 130). The resulted solution was gently stirred at room temperature until the organic phase was completely evaporated. Then, the solution was filtered through a $0.2 \mu \mathrm{m}$ syringe filter.

\section{Glucose- and methoxy-installed SPIO-polymeric micelles}

$\mathrm{N}$-hydroxysuccinimide (NHS) (20 equivalents) and 1-ethyl-3 (3-dimethyl aminopropyl) carbodiimide hydrochloride (EDC) (6.7 equivalents) were added into SPIO micelles, and the mixture was reacted for $2 \mathrm{~h}$ at room temperature. Glucosamine (15 equivalents) was then added. The $\mathrm{pH}$ of the reaction mixture was adjusted to 9 using triethylamine. The reaction was then allowed to carry out for $6 \mathrm{~h}$ at $37^{\circ} \mathrm{C}$. After that, the solution was purified by dialysis membrane with cut off MW at 50,000 Da for $24 \mathrm{~h}$. Glucose-installed SPIO-polymeric micelles (Glu-SPIO micelles) solution was then freeze dried to obtain powder for FTIR analysis. SPIO micelles without glucose were prepared similar to method mentioned above but 2-methoxyethylamine was used instead of glucosamine.

\section{Particle size determination}

Average particle size, size distribution and zeta potential of micelles were measured by dynamic light scattering (Zetasizer Nano ZS, Malvern). Micelles were diluted by water until polymer concentration was approximately $10 \mu \mathrm{g} / \mathrm{ml}$.

\section{Determination Fe content in SPIO micelles}

SPIO micelle was dissolved in $12 \mathrm{M} \mathrm{HCl}$ solution and incubated at $60{ }^{\circ} \mathrm{C}$ for $4 \mathrm{~h}$. The solution was then mixed with $1.5 \mathrm{M}$ potassium thiocyanate solution to form $\mathrm{Fe}-$ $\mathrm{SCN}$ complex. Spectrophotometer was used to measure the absorbance of each solution at the wavelength of $447 \mathrm{~nm}$.

\section{Cell culture and cytotoxicity studies}

Human prostate cancer cell line (PC-3) was cultured in Eagle's Minimum Essential Medium (EMEM) supplemented with $10 \%$ fetal bovine serum (FBS), $110 \mathrm{mg} / \mathrm{ml}$ of sodium pyruvate, $100 \mathrm{U} / \mathrm{mL}$ of penicillin and $100 \mathrm{U} / \mathrm{mL}$ of streptomycin. The culture was incubated at $5 \% \mathrm{CO}_{2}$ in a humidified atmosphere at $37{ }^{\circ} \mathrm{C}$. The cytotoxicity of SPIO micelles and Glu-SPIO micelles against human prostate cancer cells line (PC-3) was carried out by a DNA/Survival assay with various equivalent iron concentrations. Cell viability was calculated using the ratio of the number of PC-3 cells between the treated group over the untreated group (control).

\section{Cellular uptake study}

PC-3 cells at the density of $10^{6}$ cells per well in 6-well plate were incubated with the Glu-SPIO micelles and SPIO micelles for $2 \mathrm{~h}$ at the Fe concentration of 25 and $100 \mathrm{ppm}$, respectively. After incubation, cells were washed three times with PBS to remove micelles that were not taken up by cells. Cells were separated from the medium using a trypsinization method then centrifuged $\left(1500 \mathrm{rpm} 4{ }^{\circ} \mathrm{C}\right.$ for $7 \mathrm{~min}$ ). Cells were then dissolved in $12 \mathrm{M} \mathrm{HCl}$ solution to digest cells and ionize SPIO into free iron cations. The amount of Fe in PC-3 cells was determination by atomic absorption spectroscopy. 


\section{Determination of the transverse relaxivities $\left(r_{2} *\right)$}

SPIO micelles at the $\mathrm{Fe}$ concentrations between 0.18 and $0.72 \mathrm{mM}$ were mixed with $2 \%$ agarose gel then they were filled into 384 -well plate. $T_{2}$ and $1 / T_{2}{ }^{*}$ values of SPIO micelle were performed on a $3.0 \mathrm{~T}$ clinical MRI scanner (Philips, Achieva 3T MR, Netherlands, and B.V.) equipped with animal coil.

\section{In vitro MRI}

PC-3 cells at the density of $1 \times 10^{6}$ cells/well in 6-well plate were incubated with Glu-SPIO micelles or SPIO micelles at the $\mathrm{Fe}$ concentration of $100 \mathrm{ppm}$ in medium for $2 \mathrm{~h}$. Then, cells were washed with PBS, trypsinized and centrifuged at $1200 \mathrm{rpm}$ for $3 \mathrm{~min}$. Fresh PBS and $2 \%$ agarose solution were added into cells. The mixture was mixed and transferred into 384-well plate which was used as an MRI phantom. Phantom was kept at $4{ }^{\circ} \mathrm{C}$ overnight before MRI scanning. Phantom was scanned under a $3.0 \mathrm{~T}$ clinical MRI scanner equipped with wrist coil at room temperature and $1 / T_{2} *$ mapping images were acquired.

\section{Results and discussion}

\section{Characterization of carbonyl-PEG- $b$-PCL}

The synthesis of carbonyl-PEG- $b$-PCL was divided into three steps as shown in Fig. 1. First, ring opening polymerization of ethylene oxide was used to polymerize PEG using 3-benten-1-ol as an initiator. Second, the PCL block was polymerized by ring opening polymerization of $\varepsilon$ caprolactone using PEG as a macroinitiator. NMR spectrum of allyl-PEG- $b$-PCL was shown in Fig. 2 a. The
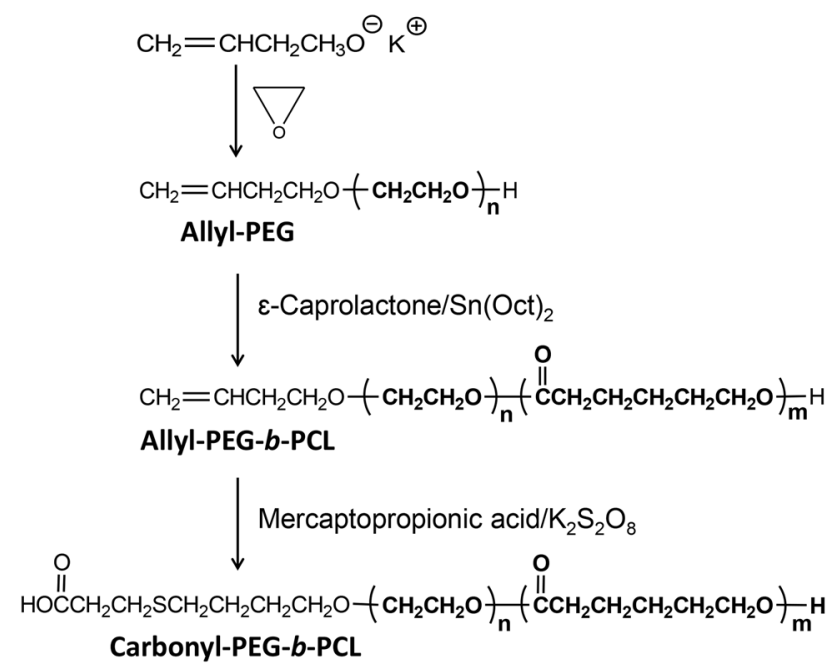

Fig. 1 Synthesis route of carbonyl-PEG- $b$-PCL multiplet peaks of allyl groups were assigned at 4.9 (peak a) $\left(\mathrm{CH}_{2}=\mathrm{CH}-\right)$ and 5.8 (peak b) $\left(\mathrm{CH}_{2}=\mathrm{CH}-\right)$ ppm. Methylene protons of PEG (-OCH $\mathrm{O}_{2} \mathrm{CH}_{2} \mathrm{O}-$ ) were assigned at $3.6 \mathrm{ppm}$ (peak c). Methylene protons of PCL peaks were assigned at 1.3 (peak f), 1.5 (peak e $+\mathrm{g}$ ), 2.3 (peak d) and 4.1(peak h) ppm at the ratio of 1:2:1:1, respectively, as shown in Fig. 2a, b. Third, the thiol-ene reaction was used to conjugate mercaptopropionic acid and allyl-PEG using AIBN as a radical initiator. The mercaptopropionic molecule in carbonyl-PEG- $b$-PCL were observed at 2.75 (peak $\mathrm{j}$, $-\mathrm{CH}_{2} \mathrm{SCH}_{2} \mathrm{CH}_{2} \mathrm{COOH}$ ), 2.55 (peak k, $-\mathrm{CH}_{2} \mathrm{SCH}_{2} \mathrm{CH}_{2}$ $\mathrm{COOH}$ ) and 2.35 (peak I, $-\mathrm{SCH}_{2} \mathrm{CH}_{2} \mathrm{COOH}$ ) ppm as shown in Fig. 2b. The molecular weight distribution of polymer was determined by GPC using PS standard. The molecular weight and DPI of allyl-PEG- $b$-PCL were $8.21 \mathrm{kDa}$ and 1.19 , respectively. For carbonyl-PEG- $b$-PCL, the molecular weight and DPI of allyl-PEG- $b$-PCL were $8.93 \mathrm{kDa}$, and 1.12 , respectively.

\section{Characterization of SPIO micelles}

The synthesis of SPIO micelles and Glu-SPIO micelles is shown in Fig. 3a. SPIO micelles were prepared by solvent evaporation method and were loaded into the core of polymeric micelles. The attachment of glucosamine molecules on the surface of SPIO micelles was carried out after micelle assembly where the carbonyl groups were present at the surface of SPIO micelles so that glucosamine molecules were completely located on the surface. The coupling agents (EDC and NHS) were used in conjugating the amine group of glucosamine and the carbonyl group of polymer by amide bond. The product was purified by dialysis method to remove excess glucosamine and coupling agents. SPIO micelles were synthesized by conjugation between 2-methoxyethylamine and carbonyl-SPIO micelles via EDC/NHS coupling agents. The conjugation of glucosamine molecules was analyzed by FTIR. Figure $3 \mathrm{~b}$ indicated the strong absorption band of the carbonyl group of ester bond in PCL chain at $1725 \mathrm{~cm}^{-1}$. Figure $3 \mathrm{c}$ indicated the new absorption band at $1650 \mathrm{~cm}^{-1}$ and was the signal from the amide bond formation between an amine group of glucose and a carboxyl of the polymer. The $-\mathrm{OH}$ stretching band at $3500 \mathrm{~cm}^{-1}$ resulted from the hydroxyl group of glucose molecules (Fig. 3c). The loading of Glu-SPIO micelles and SPIO micelles were equal to $12 \%$ because both of SPIO micelles were synthesized from the same batch of carbonyl-SPIO micelles. The size of SPIO, SPIO micelles and Glu-SPIO micelles was determined by dynamic light scattering as shown in Fig. 4a. The size of SPIO (dispersed in hexane) was $8.49 \pm 0.34 \mathrm{~nm}$. The sizes of carbonyl-SPIO micelle and Glu-SPIO micelles were $35.5 \pm 5.5$ and $35.6 \pm 4.3 \mathrm{~nm}$, respectively. Results suggest that the conjugation of glucose to SPIO micelles 
Fig. $2{ }^{1} \mathrm{H}$ NMR of allyl-PEG$b$-PCL (a) and carbonyl-PEG- $b$ PCL (b) in $\mathrm{CDCl}_{3}$

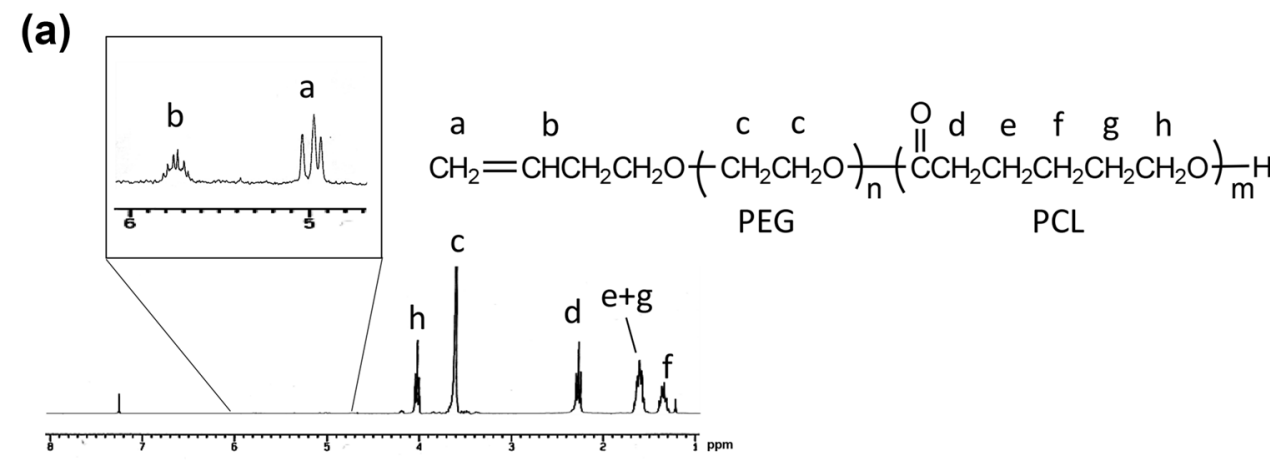

(b)

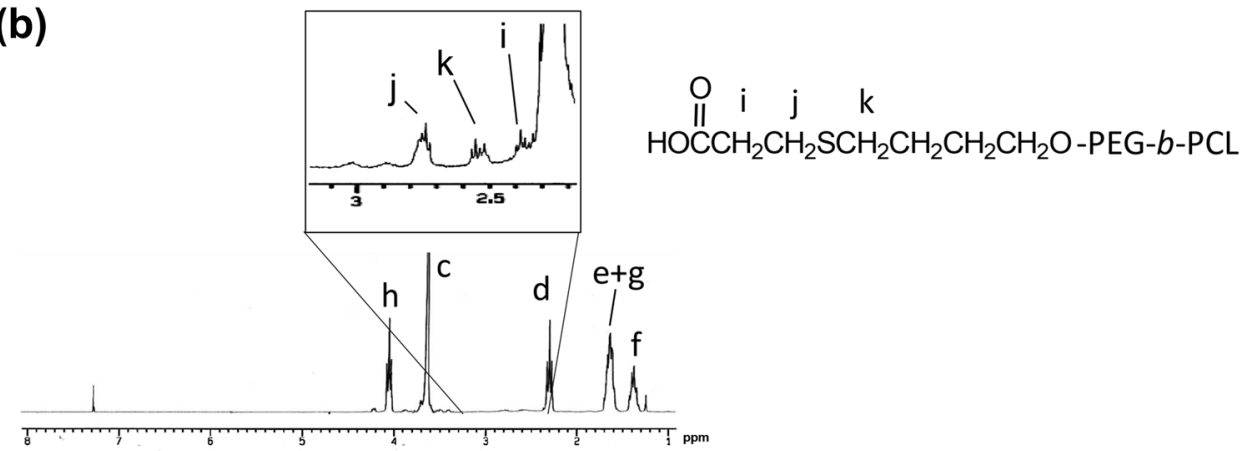

did not affect the size of micelles. However, the zeta potential of both SPIO micelles and Glu-SPIO micelles compared to carbonyl-SPIO micelles was significantly different (Table 1). The zeta potential of carbonyl-SPIO micelles had considerably negative charge $-24.3 \pm 0.4 \mathrm{mV}$ ) because of carbonyl group on the surface of micelles. The zeta potential of SPIO micelles and Glu-SPIO micelles increased to $-17.2 \pm 0.2$ and $-15.7 \pm 0.8 \mathrm{mV}$, respectively. The results also confirm the existence of glucose and methoxy group on the surface of micelles by conjugation of glucosamine and 2-methoxyethylamine to carbonyl-SPIO micelles via EDC/ NHS coupling agent.

\section{Cytotoxicity study}

The cytotoxicity of SPIO micelles and Glu-SPIO micelles against human prostate cancer cells line (PC-3) was carried out by a DNA/Survival assay with various equivalent SPIO concentrations ( $\mathrm{ppm}$ of $\mathrm{Fe}$ ). Cell viability was calculated using the ratio of the number of PC-3 cells of the treated group over the untreated group (control). From Fig. 4b, the cell viability of PC-3 cells incubated for $12 \mathrm{~h}$ with both SPIO micelles and Glu-SPIO micelles was more than $90 \%$ for all SPIO concentration proofing the biocompatibility of these micelles.

\section{Cellular uptake study}

Atomic absorption spectroscopy was employed to determine the amount of SPIO uptaked by prostate cancer cells. Interestingly, it has been noted that the cellular uptake was increased in the case when glucose molecules were attached on the surface of micelles. After incubation of cancer cells with both SPIO micelles and Glu-SPIO micelles at the $\mathrm{Fe}$ concentration of $25 \mathrm{ppm}$ for $2 \mathrm{~h}$, the iron in cancer cells was 0.326 picogram/cell while that of SPIO micelles was 0.012 picogram/cell as shown in Fig. 4c. The same result was also found when the Fe concentration was at $100 \mathrm{ppm}$ where the iron was at 0.684 and 0.133 picogram/cell for Glu-SPIO micelles and SPIO micelles, respectively. The enhancement in the uptake as a result of the targeting ligand, glucose, at the concentration of $\mathrm{Fe}$ at 25 and $100 \mathrm{ppm}$ was 27 -fold and fivefold, respectively. The difference of cell uptake between Glu-SPIO micelles and SPIO micelles of $100 \mathrm{ppm}$ was lower than $25 \mathrm{ppm}$ which was probably due to the internalization of micelles by diffusion mechanism (Mathot et al. 2007) or nonspecific uptake mechanism of micelles (Savić et al. 2003). This mechanism was pronounced at high concentration of micelles. For lower micelle concentration, micelle uptake was achieved via endocytosis. 
Fig. 3 a Schematic diagram of preparation of SPIO micelles and Glu-SPIO micelles. FTIR spectrums of carbonyl-PEG- $b$ PCL (b) and Glu-PEG- $b$-PCL (c) (a)

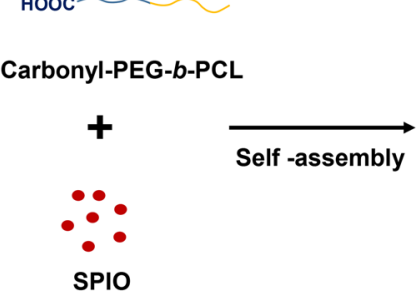

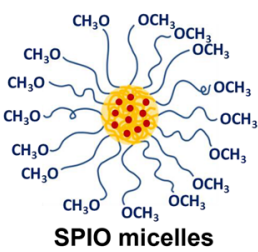

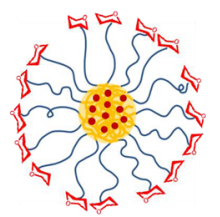

Glu-SPIO micelles

(b)

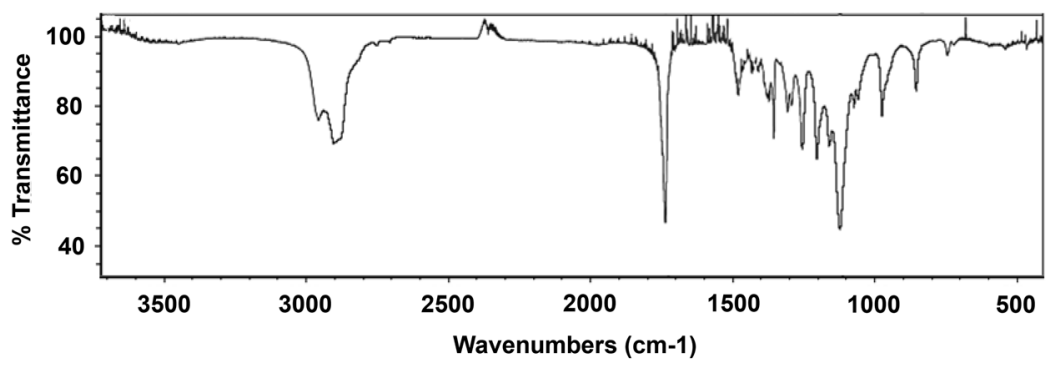

(c)

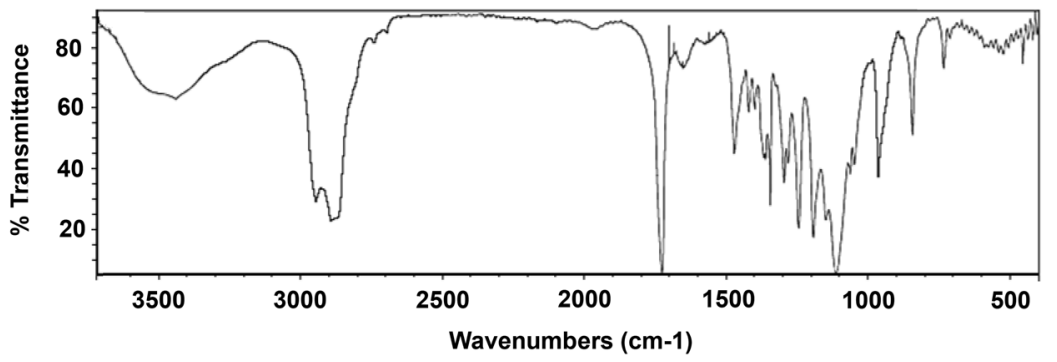

\section{Determination of the transverse relaxivities $\left(r_{2}^{*}\right)$}

After applying external magnetic field $\left(B_{0}\right)$ through the $z$ axis, magnetic moments of nuclear spins align with the direction of $B_{0}$ and produce net magnetization $\left(M_{\mathrm{z}}\right)$ in the longitudinal. $M_{z}$ can be flipped out from the original direction by irradiation of $90^{\circ}$ radio frequency (RF) pulse resulting in $M_{z}$ becoming zero. After irradiation is stopped, $M_{\mathrm{z}}$ returns spontaneously to thermal equilibrium state $\left(M_{\mathrm{z}(0)}\right)$. This process provides longitudinal relaxation times or spin-lattice relaxation $\left(T_{1}\right) . T_{1}$ relaxation time is the time $\left(M_{z}\right)$ required to return to $63 \%$ of $M_{\mathrm{z}(0)}$ and $1 / T_{1}$ is defined as $T_{1}$ relaxation rate. $T_{2}$ and $T_{2}^{*}$ is the transverse proton relaxation time, indicating decoherence of proton magnetisation because of its interaction with each other or the fluctuating magnetic moments in the surrounding. $M_{\mathrm{z}}$ is excited until $M_{\mathrm{z}}$ equals zero by $90^{\circ}$ pulse of RF irradiation. In other words, $M_{\mathrm{z}}$ is flipped into the $x y$-plane as the transverse magnetization $\left(M_{x y}\right) . M_{x y}$ is decreased due to spin-spin relaxation or $T_{2}$ relaxation. The time required for $M_{x y}$ to decrease to $37 \%$ of the maximum is defined as $T_{2}$ relaxation time and $1 / T_{2}$ is defined as $T_{2}$ relaxation rate. $T_{2}$ is generally 1000-times faster than $T_{1}$. Under external magnetic field, SPIO can produce small magnetic fields that will shorten the relaxation time $\left(T_{1}, T_{2}\right.$ and $\left.T_{2} *\right)$ of the surroundings as shown in Fig. 5b. Results show that $T_{2}{ }^{*}$ weighted images were darker when the concentration of SPIO was increased as shown in Fig. 5a. MRI sensitivity of SPIO micelles was evaluated by measuring the transversal relaxation time $\left(T_{2}^{*}\right)$ of water proton in tissue-mimicking materials (2\% agarose gel) containing SPIO micelles. $T_{2}{ }^{*}$ value was determined from exponential decay (Eq. 1), where SI is signal intensity and TE is echo time. The relaxivity $\left(r_{2}{ }^{*}\right)$ was calculated from the slope of the line which plots between $1 / T_{2} *$ relaxation rates versus $\mathrm{Fe}$ concentration as Eq. (2). 
(a)

SPIO in hexane

$8.49 \pm 0.34 \mathrm{~nm}$

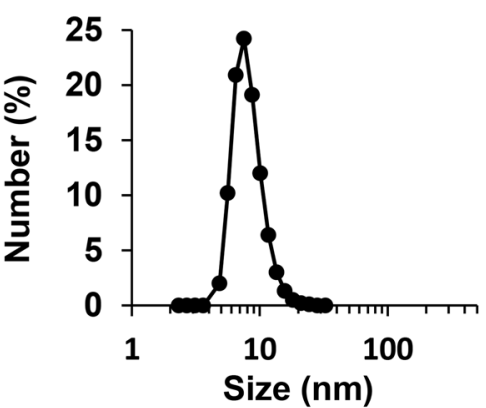

(b)

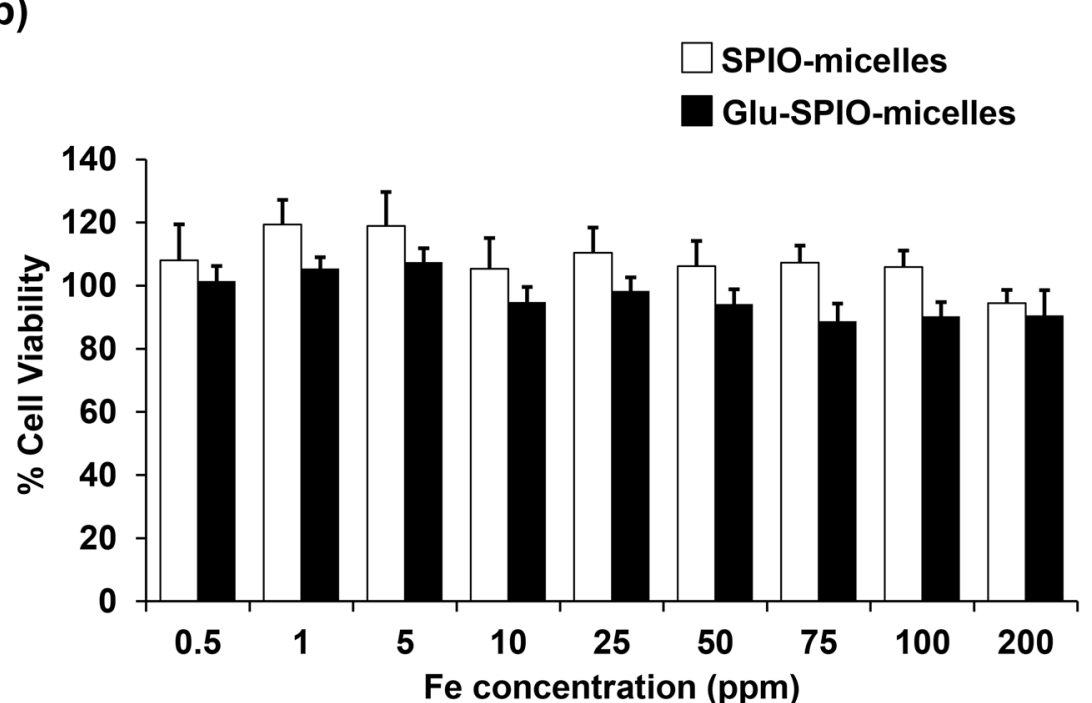

Carbonyl-SPIO-micelles

$35.0 \pm 5.5 \mathrm{~nm}$

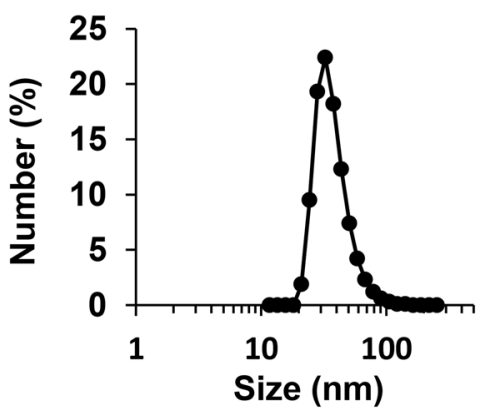

(c)

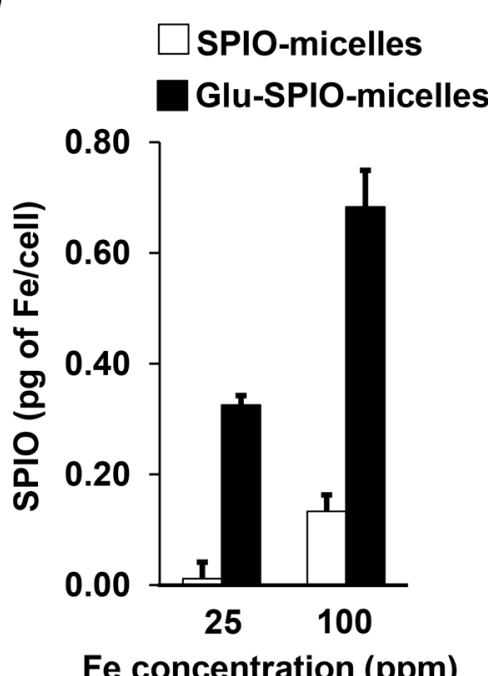

Fig. 4 a Particles size distribution of SPIO, carbonyl-SPIO micelles and Glu-SPIO micelles. b Cytotoxicity of SPIO micelle and Glu-SPIO micelles against PC-3cell after incubation for $12 \mathrm{~h}$ (mean $\pm \mathrm{SD}$; $n=6$ ). c Cellular uptake of SPIO micelles and Glu-SPIO micelles by
PC-3 cell at the Fe concentration of 25 and $100 \mathrm{ppm}$ for $2 \mathrm{~h}$ incubation (mean $\pm \mathrm{SD} ; n=2$ with each sample was measured three times)

Table 1 Properties of carbonyl-SPIO micelles, SPIO micelles and Glu-SPIO micelles

\begin{tabular}{lllll}
\hline Micelles & Surface & SPIO loading $(\% w / w)$ & Micelle size $(\mathrm{nm})$ & Zeta potential $(\mathrm{mv})$ \\
\hline $\begin{array}{l}\text { Carbonyl-SPIO } \\
\text { micelles }\end{array}$ & $-\mathrm{COOH}$ & $12.2 \%$ & $35.0 \pm 5.5$ & $-24.3 \pm 0.4$ \\
SPIO micelles & $-\mathrm{OCH}_{3}$ & $\mathrm{n} / \mathrm{a}$ & $35.4 \pm 3.7$ & $-17.2 \pm 0.2$ \\
Glu-SPIO micelles & $-\mathrm{Glucose}$ & $\mathrm{n} / \mathrm{a}$ & $35.6 \pm 4.3$ & $-15.7 \pm 0.8$ \\
\hline
\end{tabular}

$n / a$ not determined

$\mathrm{SI}_{\mathrm{TE}}=\mathrm{SI}_{\mathrm{TE}=0} \exp \left[\frac{-\mathrm{TE}}{T_{2}^{*}}\right]$

$\frac{1}{T_{2}^{*}}=\frac{1}{T_{2, H_{2} \mathrm{O}}^{*}}+r_{2}^{*}[\mathrm{Fe}]$

$T_{2}{ }^{*}$ was determined by curve fitting of exponential decay of signal intensity (SI) as a function of echo time
(Fig. 5b). $T_{2}^{*}$ values of Glu-SPIO micelles at Fe concentration of $0,0.18,0.36$ and $0.72 \mathrm{mM}$ in agarose gel were carried out by curve fitting of signal intensity decay as shown at line 1, 2, 3 and 4 in Fig. 5b, respectively. Figure $5 \mathrm{c}$ shows that $1 / T_{2} *$ value was directly corresponding to the amount of SPIO or Fe concentration. Transverse relaxivity $\left(r_{2}^{*}\right)$ was obtained from the slope of the linear regression between $1 / T_{2}^{*}$ and $\mathrm{Fe}$ concentration. The $r_{2}{ }^{*}$ 
(a)

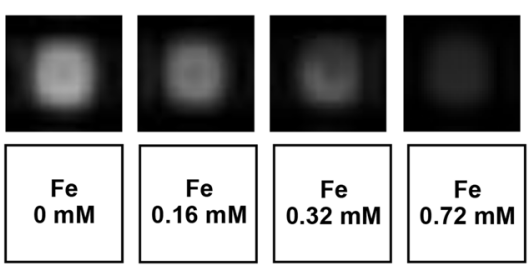

(b)

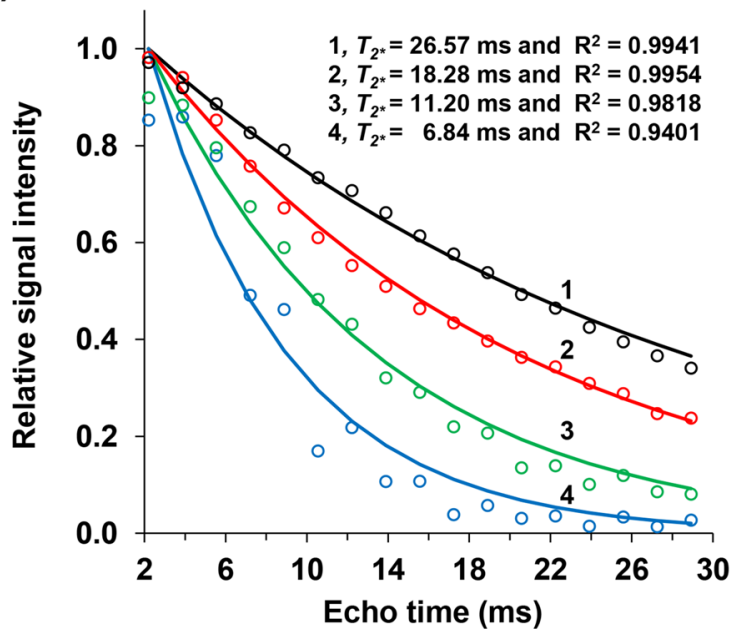

(c)

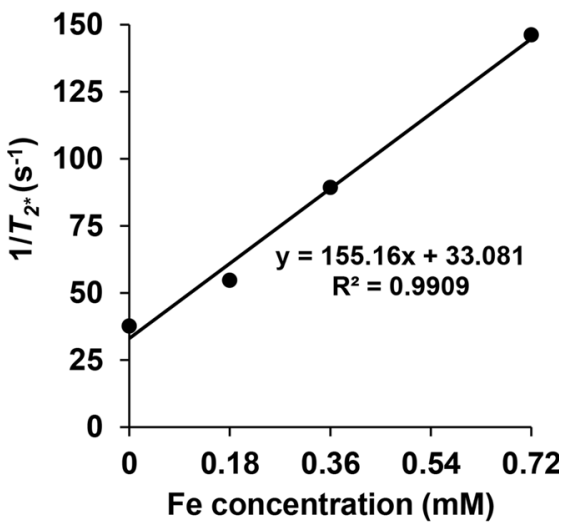

(d)

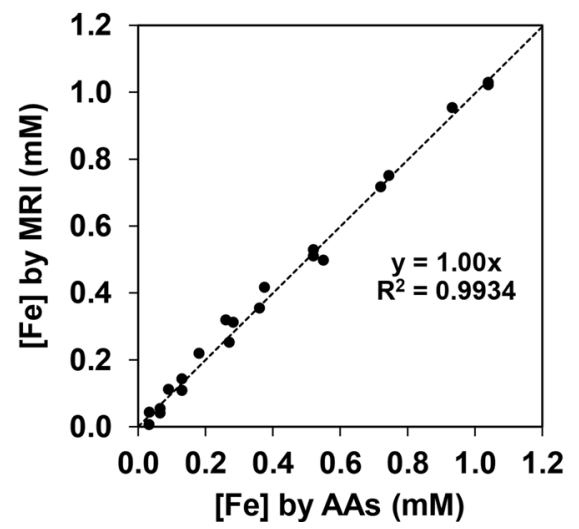

Fig. 5 a $T_{2}{ }^{*}$-weighted images $(\mathrm{TR}=50 \mathrm{~ms}$ and $\mathrm{TE}=12 \mathrm{~ms})$ of Glu-SPIO micelles at the Fe concentrations of $0,0.18,0.36$ and $0.72 \mathrm{mM}$. b Curve fitting of signal intensity as function of echo time to determine $T_{2}{ }^{*}$ of Glu-SPIO micelles at the Fe concentrations of 0

was estimated to be $155.16 \mathrm{mM}^{-1} \mathrm{~s}^{-1}$. The high relaxivity value was observed due to the clustering effect of SPIO within polymeric micelles (Nasongkla et al. 2006). To validate MRI method for the determination of iron, 21 samples of various Fe concentrations of SPIO micelles were scanned on MRI to determine $1 / T_{2} *$ value for the calculation of $\mathrm{Fe}$ concentration and compared to atomic absorption spectroscopy (AAs) method. Results of validation test was illustrated in Fig. 5d. The values of root mean square error (RMSE) and root mean absolute percentage error (MAPE) in this model was 0.026 and 9.344, respectively.

Uncertainty analysis was carried out. There are two categories of uncertainty, type $\mathrm{A}$ and type B. Type A uncertainty analysis is based on the statistical analysis of measurements as shown in Eq. (3)

$u_{\text {Type } A}=\frac{s\left(q_{k}\right)}{\sqrt{n}}$

where $u_{\text {Type } A}$ is Type A uncertainty value, $s\left(q_{k}\right)$ is standard deviation of experiment, $n$ is number of independent sample, $q_{k}$ is quantity for $\mathrm{k}=1,2,3, \ldots, n$. Type $\mathrm{B}$ (line 1), 0.18 (line 2), 0.36 (line 3) and 0.72 (line 4) mM. c $1 / T_{2} *$ as a function of iron concentration (mM) for Glu-SPIO micelles. d Validation of MRI for determination of Fe concentrations

uncertainty analysis is based on specification of instrument. Instrument uncertainty $\left(u_{I}\right)$ is divvied into two parts. First, $u_{A}$ is an error of instruments from accuracy and second, $u_{R}$ is an error of instruments from resolution. The $u_{I}$, is calculated by combining the $u_{A}$ and $u_{R}$ at $95 \%$ probability level as shown in Eq. (4)

$u_{I}=\sqrt{u_{A}^{2}+u_{R}^{2}}$

The type B uncertainty $\left(u_{\text {Type B }}\right)$ is calculated for a rectangular probability distribution at $68 \%$ probability level as shown in Eq. (5)

$u_{\mathrm{TypeB}}=\frac{u_{I}}{\sqrt{3}}$

The Eqs. (6) and (7) are combined and expanded uncertainty analysis.

$u_{\text {combine }}=\sqrt{u_{\text {Type }^{2}}+u_{\text {Type }^{2}}}$,

$u_{\text {expand }}=k \times u_{\text {combine }}$, 
where $k$ is coverage factor that is 1 for $68 \%, 2$ for $95 \%$ and 3 for $99.7 \%$ confidence levels. The uncertainty analysis and the uncertainty sources are presented in Table 2.

\section{In vitro MRI}

PC-3 cells $\left(6.4 \times 10^{5}\right.$ cells $)$ were treated with SPIO micelles and Glu-SPIO micelles at the Fe concentration of $100 \mathrm{ppm}$ for $2 \mathrm{~h}$. Then, cancer cells were harvested and mixed with $20 \mu \mathrm{L}$ of agarose and filled into the phantom which was made up of 384-well plate. $T_{2}{ }^{*}$-weighted MR images of untreated PC- 3 cells (Cell), PC- 3 cells incubated with SPIO micelles (Cell + SPIO) and PC-3 cells incubated with Glu-SPIO micelles (Cell + Glu-SPIO) were shown in Fig. 6a. The enhancement of PC-3 uptake by glucose ligand on the surface of Glu-SPIO micelles resulted in the darkening effect of MR images compared to SPIO micelles. The relative MRI signal intensity significantly decreased from 0.9 (Cell + SPIO) to 0.6 (Cell + Glu-SPIO). PC-3 cells without any treatment were used as a control group with MR intensity at 1.0. PC-3 cells treated with SPIO micelles and Glu-SPIO micelles in agaroses gel were carried out by curve fitting of signal intensity decay as shown at line 1, 2 and 3 in Fig. 6c, respectively. The summary of $T_{2} *$ and $1 / T_{2} *$ values of PC-3 cells treated with SPIO micelles and Glu-SPIO micelles is shown in Table 3. $T_{2} *$ value of cancer cells treated with Glu-SPIO micelles was significantly lower than that of cancer cells treated with SPIO micelles. Without glucose as a targeting ligand, the uptake of SPIO micelles was very low when the $T_{2}{ }^{*}$ value was similar to the control group. Unlike $T_{2}{ }^{*}$ weighted images, the $1 / T_{2}{ }^{*}$ value linearly correlated with the amount of iron per voxel in tissues, so these values can be used to quantify the amount of SPIO uptake by cancer

Table 2 Uncertainty source, combined and expanded uncertainty results

\begin{tabular}{lc}
\hline Uncertainty Source & Uncertainty $(u)$ \\
\hline $1.039 \mathrm{mM}(\mathrm{SD}=0.037$ and $n=4)$ & 0.0185 \\
MRI instrument & 2.696 \\
AAs instrument & 0.156 \\
Preparing sample & 0.144 \\
$u_{\text {combined }}(\mathrm{mM})$ & 0.0270 \\
$u_{\text {expanded }}(\mathrm{mM})$ for $68.0 \%$ confidence level & \pm 0.0270 \\
$u_{\text {expanded }}(\mathrm{mM})$ for $95.0 \%$ confidence level & \pm 0.0541 \\
$u_{\text {expanded }}(\mathrm{mM})$ for $99.7 \%$ confidence level & \pm 0.0811 \\
\hline
\end{tabular}

(a)
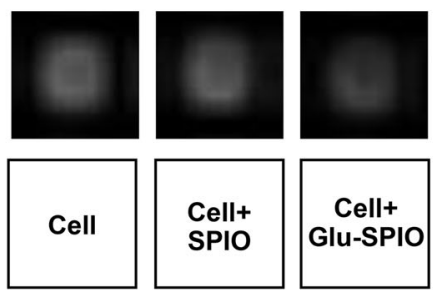

(b)

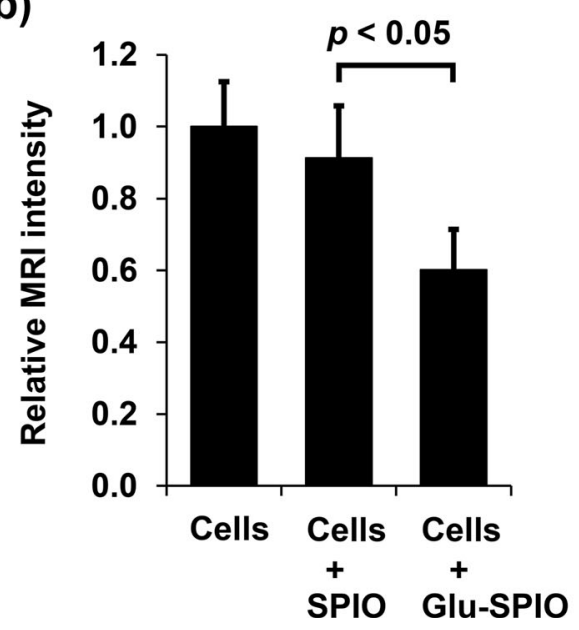

Fig. 6 a $T_{2}^{*}$-weighted images $(\mathrm{TR}=50 \mathrm{~ms}$ and $\mathrm{TE}=12 \mathrm{~ms})$ of untreated PC-3 cells, PC-3 cells treated with SPIO micelles $($ Cell + SPIO $)$ and Glu-SPIO micelles (Cell + Glu-SPIO $)$ for $2 \mathrm{~h}$ of incubation at the Fe concentration of $100 \mathrm{ppm}$. b Relative MRI (c)

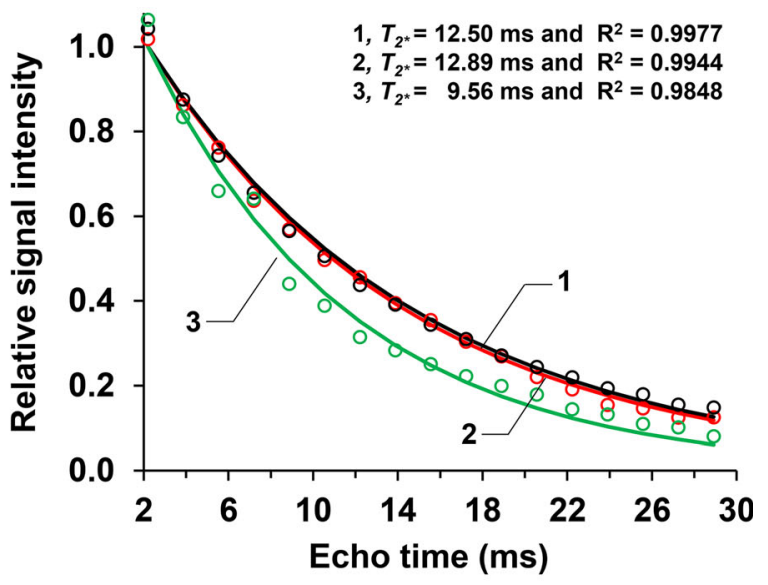

signal intensity of PC-3 cell described in Fig. 6a. c Curve fitting of signal intensity as function of echo time (line 1), PC-3 cells treated with SPIO micelles (line 2) and Glu-SPIO micelles (line 3) for $2 \mathrm{~h}$ of incubation at the Fe concentration of $100 \mathrm{ppm}$ 
Table $3 T_{2}^{*}$ and $1 / T_{2} *$ values of human prostate cancer (PC-3) cells treated with SPIO micelles and Glu-SPIO micelles at the Fe concentration of $100 \mathrm{ppm}$ for $2 \mathrm{~h}$

\begin{tabular}{lcc}
\hline Sample & $T_{2^{*}}$ Value $(\mathrm{ms})$ & $1 / T_{2^{*}}$ Value $\left(\mathrm{s}^{-1}\right)$ \\
\hline Cancer cells (control) & 12.50 & 79.99 \\
Cancer cells + SPIO micelles & 12.89 & 77.56 \\
Cancer cells + Glu-SPIO & 9.56 & 104.60 \\
$\quad$ micelles & & \\
\hline
\end{tabular}

cells. Therefore, the trend of $1 / T_{2} *$ values also confirmed the enhancement of cellular uptake by glucose ligand. Results show that $1 / T_{2} *$ of cancer cells incubated with GluSPIO micelles was higher than that of SPIO micelles by $35 \%$. These results indicated that glucose-installed SPIO micelles have potential application as MRI contrast agents to target prostate cancer cells.

\section{Conclusion}

In summary, we have developed the glucose-installed SPIO micelles as an MRI contrast agent to target cancer cells based on high-glucose metabolism. Our study demonstrated enhanced cellular uptake of SPIO micelles in human prostate cancer cell line as a result of a targeting ligand, i.e., glucose on the micelle surface. This targeting mechanism was achieved via binding of glucose to the glucose transporter on the membrane of prostate cancer cells. Glu-SPIO micelles showed high transverse relaxivities due to clustering effect of SPIO in the core of micelles. The higher $1 / T_{2} *$ values of cancer cells after treated with Glu-SPIO micelles were found when compared to SPIO micelles. Consequently, glucose can be used as a small molecule ligand to target cancer cells overexpressing glucose transporter.

Acknowledgements This research project was supported by Mahidol University, Thailand.

Open Access This article is distributed under the terms of the Creative Commons Attribution 4.0 International License (http:// creativecommons.org/licenses/by/4.0/), which permits unrestricted use, distribution, and reproduction in any medium, provided you give appropriate credit to the original author(s) and the source, provide a link to the Creative Commons license, and indicate if changes were made.

\section{References}

Blanco E, Kessinger CW, Sumer BD, Gao J (2009) Multifunctional micellar nanomedicine for cancer therapy. Exp Biol Med 234:123-131. doi:10.3181/0808-mr-250
Caster JM, Patel AN, Zhang T, Wang A (2016) Investigational nanomedicines in 2016: a review of nanotherapeutics currently undergoing clinical trials. Wiley Interdiscip Rev-Nanomed Nanobiotechnol. doi:10.1002/wnan.1416

Cheng D et al (2011) Nonclustered magnetite nanoparticle encapsulated biodegradable polymeric micelles with enhanced properties for in vivo tumor imaging. J Mater Chem 21:4796-4804. doi:10. 1039/c0jm03783d

Effert P, Beniers AJ, Tamimi Y, Handt S, Jakse G (2004) Expression of glucose transporter 1 (Glut-1) in cell lines and clinical specimens from human prostate adenocarcinoma. Anticancer Res 24:3057-3063

Hoffman JM, Gambhir SS (2007) Molecular imaging: the vision and opportunity for radiology in the future. Radiology 244:39-47. doi:10.1148/radiol.2441060773

Jin R, Lin B, Li D, Ai H (2014) Superparamagnetic iron oxide nanoparticles for MR imaging and therapy: design considerations and clinical applications. Curr Opin Pharmacol 18:18-27. doi:10.1016/j.coph.2014.08.002

Jokerst JV, Lobovkina T, Zare RN, Gambhir SS (2011) Nanoparticle PEGylation for imaging and therapy. Nanomedicine 6:715-728

Liu H, Zhang J, Chen X, Du XS, Zhang JL, Liu G, Zhang WG (2016) Application of iron oxide nanoparticles in glioma imaging and therapy: from bench to bedside. Nanoscale 8:7808-7826. doi:10. 1039/c6nr00147e

Luciani A et al (2004) Glucose-receptor MR imaging of tumors: study in mice with PEGylated paramagnetic niosomes. Radiology 231:135-142. doi:10.1148/radiol.2311021559

Mathot F, Schanck A, Van Bambeke F, Ariën A, Noppe M, Brewster M, Préat V (2007) Passive diffusion of polymeric surfactants across lipid bilayers. J Control Release 120:79-87. doi:10.1016/ j.jconrel.2007.03.015

Movassaghian S, Merkel OM, Torchilin VP (2015) Applications of polymer micelles for imaging and drug delivery. Interdiscip RevNanomed Nanobiotechnol 7:691-707. doi:10.1002/wnan.1332

Nasongkla N, Shuai X, Ai H, Weinberg BD, Pink J, Boothman DA, Gao J (2004) cRGD-functionalized polymer micelles for targeted doxorubicin delivery. Angew Chem-Int Edit 43:6323-6327. doi:10.1002/anie. 200460800

Nasongkla $\mathrm{N}$ et al (2006) Multifunctional polymeric micelles as cancer-targeted, MRI-ultrasensitive drug delivery systems. Nano Lett 6:2427-2430. doi:10.1021/n1061412u

Puntawee S, Theerasilp M, Reabroi S, Saeeng R, Piyachaturawat P, Chairoungdua A, Nasongkla N (2016) Solubility enhancement and in vitro evaluation of PEG- $b$-PLA micelles as nanocarrier of semi-synthetic andrographolide analogue for cholangiocarcinoma chemotherapy. Pharm Dev Technol 21:437-444. doi:10. 3109/10837450.2015.1016619

Savić R, Luo L, Eisenberg A, Maysinger D (2003) Micellar nanocontainers distribute to defined cytoplasmic organelles. Science 300:615-618. doi:10.1126/science.1078192

Sheikholeslami M (2017a) Influence of magnetic field on nanofluid free convection in an open porous cavity by means of Lattice Boltzmann method. J Mol Liq 234:364-374. doi:10.1016/j. molliq.2017.03.104

Sheikholeslami M (2017b) Magnetic field influence on nanofluid thermal radiation in a cavity with tilted elliptic inner cylinder. J Mol Liq 229:137-147. doi:10.1016/j.molliq.2016.12.024

Sheikholeslami M (2017c) Magnetohydrodynamic nanofluid forced convection in a porous lid driven cubic cavity using Lattice Boltzmann method. J Mol Liq 231:555-565. doi:10.1016/j. molliq.2017.02.020

Sun S, Zeng H, Robinson DB, Raoux S, Rice PM, Wang SX, Li G (2004) Monodisperse MFe2O4 ( $\mathrm{M}=\mathrm{Fe} \mathrm{Co}, \mathrm{Mn})$ nanoparticles. J Am Chem Soc 126:273-279 
Theerasilp M, Nasongkla N (2013) Comparative studies of poly(€caprolactone) and poly(D, L-lactide) as core materials of polymeric micelles. J Microencapsul 30:390-397. doi:10.3109/ 02652048.2012.746746

Theerasilp M, Chalermpanapun P, Ponlamuangdee K, Sukvanitvichai D, Nasongkla N (2017) Imidazole-modified deferasirox encapsulated polymeric micelles as $\mathrm{pH}$-responsive iron-chelating nanocarrier for cancer chemotherapy. RSC Adv 7:11158-11169. doi:10.1039/c6ra26669j

Venturelli L et al (2016) Glucose is a key driver for GLUT1-mediated nanoparticles internalization in breast cancer cells. Sci Rep. doi:10.1038/srep21629

Weissleder R, Pittet MJ (2008) Imaging in the era of molecular oncology. Nature 452:580-589. doi:10.1038/nature06917

Xiong F, Zhu Z-Y, Xiong C, Hua X-Q, Shan X-H, Zhang Y, Gu N (2012) Preparation, characterization of 2-deoxy-D-glucose functionalized dimercaptosuccinic acid-coated maghemite nanoparticles for targeting tumor cells. Pharm Res 29:1087-1097. doi:10.1007/s11095-011-0653-9

Zhang C, Xie X, Liang S, Li M, Liu Y, Gu H (2012) Mono-dispersed high magnetic resonance sensitive magnetite nanocluster probe for detection of nascent tumors by magnetic resonance molecular imaging. Nanomed-Nanotechnol Biol Med 8:996-1006. doi:10. 1016/j.nano.2011.11.013

\section{Publisher's Note}

Springer Nature remains neutral with regard to jurisdictional claims in published maps and institutional affiliations. 